\title{
Effect of Organic Mixtures on Stone Germination and Seedling Growth of Mango (Mangifera indica) cv. Totapuri under Nethouse and Polyhouse Conditions
}

\author{
S. Vandana, P. Venkatesha Murthy and Balesh Goudappanavar* \\ Department of Horticulture, College of Agriculture, \\ University of Agricultural Sciences, Bengaluru-560065, India \\ *Corresponding author
}

\begin{tabular}{l} 
K e y w o r d s \\
$\begin{array}{l}\text { Organic mixtures, } \\
\text { PGPR, Quality, } \\
\text { Stone germination }\end{array}$ \\
Article Info \\
\hline $\begin{array}{l}\text { Accepted: } \\
12 \text { March } 2020 \\
\text { Available Online: } \\
\text { 10 April } 2020\end{array}$ \\
\hline
\end{tabular}

\section{A B S T R A C T}

\section{Introduction}

Mango is well known fruit crop and belongs to Anacardiaceae family. It is one of the popular fruits among the masses in the universe. It is called as "King of tropical fruits". India has the richest collection and wide cultivation. The genus Mangifera contains more than 42 species. $M$. indica is commercially cultivated species. It is indigenous to North-East India and North Burma, in the foot hills of the Himalayas, and is said to have originated in the Indo-Burma region (De Candolle, 1904). 
The rootstocks play very important role in fruit production. The height and vigour of plants, yield, size, maturity, colour, storability of fruits, the disease resistance, salt tolerance and adverse weather conditions are influenced by rootstocks (Samaddar and Chakrabarti, 1988).

Proper media with organic mixtures like vermicompost, cocopeat, beneficial plant growth promoting rhizobacteria (Microbial consortium) will influence on germination, growth of seedlings. Use of nethouse and polyhouse for production of nursery plants in fruit crops is being practiced as a new technology to know their effects on germination and the growth of plants under nursery condition. Keeping the above factors in view, the present study was under taken with different organic mixtures for better stone germination of mango stones under nethouse and polyhouse condition.

\section{Materials and Methods}

Experiment on "Effect of organic mixtures on stone germination and seedling growth of mango (Mangiferaindica L.) cv. Totapuri under nethouse and polyhouse conditions" were carried out at Department of Horticulture, University of Agricultural Sciences, G.K.V.K, Bengaluru during the year 2018-19.

The experiment was tested in Complete Randomized Design (CRD) with three replications and consisted of ten treatments namely $\mathrm{T}_{1}-$ Soil: Sand: FYM [2:1:1] (Control), $\mathrm{T}_{2}$ - Soil: Sand: Vermicompost [2:1:1], $\mathrm{T}_{3}$ - Soil: Sand: Cocopeat [2:1:1], $\mathrm{T}_{4}$ - Soil: Sand: FYM: Vermicompost [2:1:1:1], $\mathrm{T}_{5}$ - Soil: Sand: FYM: Cocopeat [2:1:1:1], $\mathrm{T}_{6}$ - Soil: Sand: FYM: Vermicompost: Cocopeat [2:1:1:1:1], $\mathrm{T}_{7}$ - Soil: Sand: FYM: PGPR [2:1:1:1], $\mathrm{T}_{8}$ - Soil: Sand: Vermicompost: PGPR [2:1:1:1], $\mathrm{T}_{9}$ - Soil: Sand: Cocopeat:
PGPR [2:1:1:1], $\mathrm{T}_{10}$ - Soil: Sand: FYM: Vermicompost: Cocopeat: PGPR $[2: 1: 1: 1: 1: 1]$.

Observation on stone germination in mango from each treatment were recorded separately for each day after sowing and expressed in number of days. Germination of seedlings were recorded at two days intermission initiation from the date of sowing to till the germination had totally ceased and expressed as per cent of germination.

Germination $(\%)=\frac{\text { Total no.of stones germinated }}{\text { Total no.of stones sown }} \times 100$

Statistical analysis was estimated by analysis of variance as per the technique given by Fisher (1963). Data has been suitably presented in the form of tables.

Observations are recorded on Observation on plant height, number of leaves and stem girth, recorded at 60, 90, 120,150, 180 days after sowing. The plant height was recorded by quantifying of five randomly culled plants from the base to the growing tip and stem girth of papaya was measured $1 \mathrm{~cm}$ above the media surface for all the five randomly selected plants. Statistical analysis was estimated by analysis of variance as per the technique given by Fisher (1963). Data has been suitably presented in the form of tables and graphs.

\section{Results and Discussion}

\section{Stone germination}

\section{Number of days taken for stone germination}

The data on days taken for stone germination as influenced by different organic mixtures under polyhouse and nethouse condition are presented in Table 1. 
Under polyhouse conditions, the treatment $\left(\mathrm{T}_{10}\right)$ consists of Soil: Sand: FYM: Vermicompost: Cocopeat: PGPR [2:1:1:1:1:1] recorded early stone germination (17.79 days). The longest period (24.38 days) was perceived the stone germination in control $\left(\mathrm{T}_{1}\right)$ consists of Soil: Sand: FYM [2:1:1].

Similarly, under nethouse condition the significant differences were recorded among the treatments. The treatment $\left(\mathrm{T}_{10}\right)$ combination consists of Soil: Sand: FYM: Vermicompost: Cocopeat: PGPR [2:1:1:1:1:1] recorded early stone germination (20.88 days) and longer period (26.08 days) of stone germination was taken in control.

The combination of organic mixtures like cocopeat, vermicompost in combination of microbial consortium (which contains plant growth promoting rhizobacteria) influenced the early germination with good physical and biological conditions.

Media combination with vermicompost and cocopeat shows positive effect on germination, probably owing to a synergistic combination of both these factors can influence positively by improving physical condition of the media and supply of nutritional factors (Sahni et al., 2007) in cashew.

The production of plant growth promoting substances by plant growth promoting microbes which can enhances cell division and root development, higher nitrogen fixation by nitrogen fixing bacteria, phosphorous and other nutrients fixing bacteria were responsible for early germination, healthy and quality seedlings.

Jayashree and Jagadeesh (2017) in vegetable seedlings and Venkata Subbaiah et al., (2018) in brinjal.

\section{Number of days taken for 50 per cent of germination}

Under polyhouse conditions, significant differences were observed among the treatments with respect to number of days taken for 50 per cent of germination (Table 1).

The minimum days taken for 50 per cent germination (20.00 days) was noted in treatment $\left(\mathrm{T}_{10}\right)$ consists of Soil: Sand: FYM: Vermicompost: Cocopeat: PGPR [2:1:1:1:1:1]. Whereas, maximum days required to reach 50 per cent germination (30.67 days) was observed in control treatment $\left(\mathrm{T}_{1}\right)$ consist of Soil: Sand: FYM $[2: 1: 1]$.

Similarly, under nethouse conditions significant differences were noticed among the treatments with respect to number of days taken for 50 per cent of germination (Table 2).

The minimum days taken for 50 per cent germination (22.67 days) was recorded in treatment $\left(\mathrm{T}_{10}\right)$ combination with Soil: Sand: FYM: Vermicompost: Cocopeat: PGPR [2:1:1:1:1:1], whereas maximum days required to reach 50 per cent germination (34.33 days) was observed in control.

However, the different combination of organic mixtures added to the media may have enhanced the stone germination percentage with all combined media treatment. This may be due to combination of organic mixtures helps in nutrient and water uptake and also maintenance of good physical and chemical properties of the media.

The results were in conformity with the observation made by Geetha et al., (2007) in mango and Parmeswari et al., (2001) in tamarind. 


\section{Germination percentage}

The combination of organic mixtures showed significant differences among the treatments with respect to germination percentage (Table 1). The highest germination percentage $(80.00 \%)$ was recorded in treatment $\left(\mathrm{T}_{10}\right)$ combined with Soil: Sand: FYM: Vermicompost: Cocopeat: PGPR [2:1:1:1:1:1] and minimum germination percentage $(46.67$ $\%$ ) was observed in control treatment $\left(\mathrm{T}_{1}\right)$ under polyhouse condition. Similarly, under nethouse conditions the highest germination percentage $(70.00 \%)$ was recorded in treatment $\left(\mathrm{T}_{10}\right)$ consists of Soil: Sand: FYM: Vermicompost: Cocopeat: PGPR [2:1:1:1:1:1] and minimum germination percentage $(43.33 \%)$ was recorded in control.

The similar trends are in line with Jayant Raman (2012) where, increased germination due to application of biofertilizers i.e. Azotobacterin apple. The same results was supported by Sinish et al., (2005) in cashew and Pathak et al., (2013) in guava.

\section{Plant height (cm)}

The impacts of organic mixtures on plant height at different stages of seedling growth are presented in Table 2 and Figure 1.

The plant height of mango was significantly influenced with age of seedlings at different media combination treatments. According to age of the plant, the seedling height increased in all the treatments.

But, the rate of increase in plant height varied among the treatments, the higher plant height was noticed in treatment $\left(\mathrm{T}_{10}\right)$ consists of Soil: Sand: FYM: Vermicompost: Cocopeat: PGPR [2:1:1:1:1:1] as compared to control treatment $\left(\mathrm{T}_{1}\right)$. With increase in DAS plant height increased both in polyhouse and nethouse condition in all the treatments. At any given stage, in particular at 180 DAS, the plant height was superior $(55.07 \mathrm{~cm})$ in treatment $\left(\mathrm{T}_{10}\right)$ combined with Soil: Sand: FYM: Vermicompost: Cocopeat: PGPR [2:1:1:1:1:1]. Whereas, the minimum plant height was noted $(38.62 \mathrm{~cm})$ in control treatment $\left(\mathrm{T}_{1}\right)$ contains Soil: Sand: FYM [2:1:1] under polyhouse conditions. Similarly, under nethouse conditions the plant height was recorded maximum $(44.81 \mathrm{~cm})$ in treatment $\left(\mathrm{T}_{10}\right)$ with Soil: Sand: FYM: Vermicompost: Cocopeat: PGPR [2:1:1:1:1:1] Whereas, minimum plant height $(27.91 \mathrm{~cm})$ was recorded in control treatment $\left(\mathrm{T}_{1}\right)$ at 180 DAS..

Vermicompost provides adequate nutrients and enhances both physical properties and water holding capacity. Combined application of vermicompost and cocopeat has showed significant effect on plant height and seedling growth. The plant growth promoting rhizobacteria in the media helps in fixation of nitrogen and makes it available to the plant. Sahni et al., (2007) opined that, performance of plant growth promoting rhizobacteria is enhanced by vermicompost in cashew.

\section{Number of leaves per plant}

The data on number of leaves recorded per plant at 60, 90, 120, 150, 180 DAS are presented in Table 3.

The number of leaves per plant increased with the age of plants in all treatments. Highest number of leaves per plant (19.75) was found in treatment $\left(\mathrm{T}_{10}\right)$ combination with Soil: Sand: FYM: Vermicompost: Cocopeat: PGPR [2:1:1:1:1:1] at 180 DAS.

However, the lowest number of leaves per plant (13.50) was in control treatment $\left(\mathrm{T}_{1}\right)$, where only basal media is used under polyhouse conditions. Similarly, under nethouse conditions the number of leaves per 
plant increased with the age of plants in all treatments. Highest number of leaves per plant (14.00) was found in treatment $\left(\mathrm{T}_{10}\right)$ combined with Soil: Sand: FYM: Vermicompost: Cocopeat: PGPR [2:1:1:1:1:1] at 180 DAS. The lowest number of leaves per plant (10.17) was registered in control treatment under nethouse conditions.

More number of leaves in combination of treatments may be due to better nutrient availability leading to higher production of photosynthetically functional leaves and better girth of the seedlings due to growing media reported by SukhjitKaur (2017) in mango.

\section{Stem diameter (cm)}

The stem diameter of mango seedling recorded at 60, 90, 120, 150 and 180 DAS are presented in Table 4. Significantly the highest stem diameter $(1.07 \mathrm{~cm})$ was found in treatment $\left(\mathrm{T}_{10}\right)$ combination with Soil: Sand: FYM: Vermicompost: Cocopeat: PGPR [2:1:1:1:1:1] and the lowest stem diameter $(0.78 \mathrm{~cm})$ was observed in control treatment $\left(\mathrm{T}_{1}\right)$ under polyhouse conditions at 180 DAS.

Significantly highest stem diameter $(0.92 \mathrm{~cm})$ was found in treatment $\left(\mathrm{T}_{10}\right)$ with Soil: Sand: FYM: Vermicompost: Cocopeat: PGPR [2:1:1:1:1:1] which was followed by treatment $\mathrm{T}_{9}(0.89 \mathrm{~cm})$ with Soil: Sand: Cocopeat: PGPR [2:1:1:1] and the lowest stem diameter $(0.70 \mathrm{~cm})$ was observed in control treatment $\left(\mathrm{T}_{1}\right)$ under nethouse conditions at $180 \mathrm{DAS}$.

Media with microbial consortium results, higher plant growth and stem diameter may be due to production of plant growth promoting substances (which are known to cause enhanced cell division and root development) by rhizobacteria (Jayashree et al., 2017). The result on seedling diameter was influenced by media and biofertilizers. The reason for the best performance due to good drainage qualities of the medium (Chiranjeevi et al., 2018) in aonla seedlings.

\section{Vigour index of fresh weight and dry weight at 180 DAS}

The data on vigour index of fresh weight is presented in Table 5 The highest vigour index of fresh weight (3822.00) was found in treatment $\left(\mathrm{T}_{10}\right)$ consists of Soil: Sand: FYM: Vermicompost: Cocopeat: PGPR [2:1:1:1:1:1] at 180 DAS and the lowest vigour index of fresh weight (1649.50) was obtained from control treatment $\left(\mathrm{T}_{1}\right)$ which contains basal media under polyhouse conditions.

Similarly, under nethouse conditions, the highest vigour index of fresh weight (3051.60) was found in treatment $\left(\mathrm{T}_{10}\right)$ consists of Soil: Sand: FYM: Vermicompost: Cocopeat: PGPR [2:1:1:1:1:1] at 180 DAS and the lowest vigour index of fresh weight (1355.57) was obtained from control treatment $\left(\mathrm{T}_{1}\right)$.

The highest vigour index of dry weight (2854.60) was found in treatment $\left(\mathrm{T}_{10}\right)$ consists of Soil: Sand: FYM: Vermicompost: Cocopeat: PGPR [2:1:1:1:1:1] which was followed by $\mathrm{T}_{9}$ (2458.50) with Soil: Sand: Cocopeat: PGPR [2:1:1:1] and the lowest vigour index of dry weight (1099.00) was obtained from control treatment $\left(\mathrm{T}_{1}\right)$ which contains only basal media under polyhouse condition at $180 \mathrm{DAS}$.

Similarly, under nethouse conditions, the highest vigour index of dry weight (2286.43) was found in treatment $\left(\mathrm{T}_{10}\right)$ combined with Soil: Sand: FYM: Vermicompost: Cocopeat: PGPR [2:1:1:1:1:1] at 180 DAS and the lowest vigour index of dry weight(917.73) was obtained from control treatment $\left(\mathrm{T}_{1}\right)$. 
Table.1 Effect of organic mixtures on stone germination and germination percentage of Mango Cv. Totapuri under polyhouse and nethouse conditions

\begin{tabular}{|c|c|c|c|c|c|c|}
\hline \multirow{2}{*}{ Treatment } & \multicolumn{3}{|c|}{ Polyhouse } & \multicolumn{3}{|c|}{ Nethouse } \\
\hline & $\begin{array}{c}\text { Days taken for } \\
\text { germination }\end{array}$ & $\begin{array}{c}\text { Days taken for } \\
50 \text { per cent } \\
\text { germination }\end{array}$ & $\begin{array}{c}\text { Germination } \\
\text { percentage }\end{array}$ & $\begin{array}{c}\text { Days taken for } \\
\text { germination }\end{array}$ & $\begin{array}{c}\text { Days taken for } \\
50 \text { per cent } \\
\text { germination }\end{array}$ & $\begin{array}{c}\text { Germination } \\
\text { percentage }\end{array}$ \\
\hline $\mathbf{T}_{1}$ & 24.38 & 30.67 & 46.67 & 26.08 & 34.33 & 43.33 \\
\hline $\mathbf{T}_{2}$ & 24.24 & 29.67 & 50.00 & 25.20 & 31.67 & 46.67 \\
\hline $\mathbf{T}_{\mathbf{3}}$ & 24.02 & 29.33 & 60.00 & 24.55 & 29.00 & 56.67 \\
\hline $\mathbf{T}_{4}$ & 24.08 & 29.67 & 53.33 & 26.41 & 33.67 & 53.33 \\
\hline $\mathbf{T}_{5}$ & 23.21 & 24.67 & 63.33 & 23.67 & 24.67 & 56.67 \\
\hline $\mathbf{T}_{6}$ & 23.84 & 26.00 & 63.33 & 25.49 & 27.33 & 53.33 \\
\hline $\mathbf{T}_{7}$ & 23.36 & 26.00 & 56.67 & 23.55 & 24.00 & 63.33 \\
\hline $\mathbf{T}_{8}$ & 20.85 & 25.00 & 66.67 & 23.29 & 27.00 & 60.00 \\
\hline $\mathbf{T}_{9}$ & 19.58 & 22.67 & 73.33 & 21.82 & 23.67 & 63.33 \\
\hline $\mathbf{T}_{10}$ & 17.79 & 20.00 & 80.00 & 20.88 & 22.67 & 70.00 \\
\hline F-test & $*$ & $*$ & $*$ & $*$ & $*$ & $*$ \\
\hline S.Em \pm & 0.58 & 0.97 & 3.33 & 0.94 & 1.94 & 3.94 \\
\hline CD@5\% & 1.71 & 2.85 & 9.83 & 2.78 & 5.71 & 11.63 \\
\hline $\begin{array}{l}{ }^{*} \text { Significant at } 5 \\
T_{1}-\text { Soil: Sand: } \\
T_{2}-\text { Soil: Sand: } \\
T_{3}-\text { Soil: Sand: } \\
T_{4}-\text { Soil: Sand: } \\
T_{5}-\text { Soil: Sand: }\end{array}$ & $\begin{array}{l}\text { NS - Non Significar } \\
\text { M [2:1:1] (Control) } \\
\text { micompost }[2: 1: 1] \\
\text { opeat }[2: 1: 1] \\
\text { M: Vermicompost }[2 \\
\text { M: Cocopeat }[2: 1: 1: 1\end{array}$ & $\begin{array}{l}\text { DAS - Days af } \\
\mathrm{T}_{6}-\text { Soil: Sand } \\
\mathrm{T}_{7}-\text { Soil: Sand } \\
\mathrm{T}_{8}-\text { Soil: Sand: } \\
\mathrm{T}_{9}-\text { Soil: Sand } \\
\mathrm{T}_{10}-\text { Soil: Sanc }\end{array}$ & $\begin{array}{l}\text { ng Where, PGP } \\
\text { Vermicompost: } \\
\text { PGPR [2:1:1:1] } \\
\text { compost: PGPR [ } \\
\text { eat: PGPR [2:1:1 } \\
\text { Vermicompost: }\end{array}$ & $\begin{array}{l}\text { licrobial consortium } \\
\text { peat }[2: 1: 1: 1: 1] \\
\text { 1] } \\
\text { peat: PGPR }[2: 1: 1: 1\end{array}$ & $\mathrm{ml} /$ polybag) & \\
\hline
\end{tabular}


Table.2 Effect of different organic mixtures on plant height $(\mathrm{cm})$ at different stages of growth of seedlings under polyhouse and nethouse conditions

\begin{tabular}{|c|c|c|c|c|c|c|c|c|c|c|}
\hline \multirow{3}{*}{ Treatment } & \multicolumn{10}{|c|}{ Plant Height(cm) } \\
\hline & \multicolumn{5}{|c|}{ Polyhouse } & \multicolumn{5}{|c|}{ Nethouse } \\
\hline & 60DAS & 90DAS & 120DAS & 150DAS & 180DAS & 60DAS & 90DAS & 120DAS & 150DAS & 180DAS \\
\hline $\mathbf{T}_{1}$ & 17.49 & 21.89 & 24.16 & 28.55 & 38.62 & 15.04 & 16.41 & 21.78 & 22.95 & 27.91 \\
\hline $\mathbf{T}_{2}$ & 18.55 & 23.87 & 25.05 & 30.51 & 39.72 & 16.44 & 17.47 & 22.39 & 23.86 & 33.35 \\
\hline $\mathbf{T}_{\mathbf{3}}$ & 19.93 & 22.46 & 25.35 & 29.1 & 40.99 & 17.39 & 19.17 & 22.25 & 23.67 & 31.66 \\
\hline $\mathbf{T}_{4}$ & 19.55 & 22.93 & 25.04 & 29.32 & 39.67 & 18.01 & 19.63 & 22.49 & 25.59 & 32.40 \\
\hline $\mathbf{T}_{5}$ & 19.99 & 23.46 & 25.35 & 30.09 & 40.87 & 18.63 & 20.09 & 22.19 & 25.71 & 32.27 \\
\hline $\mathbf{T}_{6}$ & 20.23 & 23.09 & 26.41 & 31.70 & 41.28 & 18.93 & 20.28 & 22.98 & 26.16 & 35.23 \\
\hline $\mathbf{T}_{7}$ & 20.86 & 24.60 & 26.99 & 33.24 & 43.05 & 19.48 & 20.65 & 24.35 & 27.82 & 37.60 \\
\hline $\mathbf{T}_{8}$ & 20.92 & 23.62 & 26.55 & 34.38 & 48.74 & 20.1 & 21.08 & 23.63 & 26.11 & 38.69 \\
\hline $\mathbf{T}_{9}$ & 22.35 & 25.32 & 33.39 & 43.21 & 48.46 & 20.39 & 22.38 & 25.09 & 33.68 & 43.97 \\
\hline $\mathbf{T}_{10}$ & 23.14 & 26.02 & 34.33 & 44.43 & 55.07 & 20.86 & 23.25 & 26.67 & 34.62 & 44.81 \\
\hline F-test & $*$ & $*$ & $*$ & $*$ & $*$ & $*$ & $*$ & $*$ & $*$ & $*$ \\
\hline SEm \pm & 0.58 & 0.37 & 1.08 & 1.84 & 1.04 & 0.38 & 0.60 & 0.62 & 0.54 & 2.47 \\
\hline CD@5\% & 1.69 & 1.09 & 3.18 & 5.42 & 3.06 & 1.128 & 1.78 & 1.84 & 1.61 & 7.28 \\
\hline $\begin{array}{l}* \text { Significant at } 5 \\
\mathrm{~T}_{1}-\text { Soil: Sand: } \\
\mathrm{T}_{2}-\text { Soil: Sand: } \\
\mathrm{T}_{3} \text { - Soil: Sand: } \\
\mathrm{T}_{4} \text { - Soil: Sand: } \\
\mathrm{T}_{5}-\text { Soil: Sand: }\end{array}$ & $\begin{array}{l}\text { NS - Non } \\
\text { M [2:1:1] } \\
\text { rmicompos } \\
\text { copeat }[2: 1 \\
\text { M: Vermic } \\
\text { M: Cocope }\end{array}$ & $\begin{array}{l}\text { nificant } \\
\text { trol) } \\
1: 1] \\
\text { ost }[2: 1: 1: 1] \\
2: 1: 1: 1]\end{array}$ & $\begin{array}{l}\text { DAS - Days } \\
\mathrm{T}_{6}-\text { Soil: } \mathrm{Sa} \\
\mathrm{T}_{7}-\text { Soil: } \mathrm{Sa} \\
\mathrm{T}_{8}-\text { Soil: } \mathrm{Sar} \\
\mathrm{T}_{9}-\text { Soil: } \mathrm{Sa} \\
\mathrm{T}_{10}-\text { Soil: } \mathrm{S}\end{array}$ & $\begin{array}{l}\text { ter sowing } \\
\text { FYM: Vern } \\
\text { FYM: PGP } \\
\text { Vermicomp } \\
\text { Cocopeat: P } \\
\text { : FYM: Ver }\end{array}$ & $\begin{array}{l}\text { Where } \\
\text { compost: Co } \\
{[2: 1: 1: 1]} \\
\text { t: PGPR [2: } \\
\text { PR [2:1:1:1] } \\
\text { icompost: C }\end{array}$ & $\begin{array}{l}\text { GPR }- \text { Mic } \\
\text { peat }[2: 1: 1:\end{array}$ & ial consorti & $\mathrm{n}(25 \mathrm{ml} / \mathrm{po}$ & & \\
\hline
\end{tabular}


Table.3 Effect of different organic mixtures on number of leaves per plant at different stages of growth of seedlings under polyhouse and nethouse conditions

\begin{tabular}{|c|c|c|c|c|c|c|c|c|c|c|}
\hline \multirow{3}{*}{ Treatment } & \multicolumn{10}{|c|}{ Number of leaves per plant } \\
\hline & \multicolumn{5}{|c|}{ Polyhouse } & \multicolumn{5}{|c|}{ Nethouse } \\
\hline & 60DAS & 90DAS & 120DAS & 150DAS & 180DAS & 60DAS & 90DAS & 120DAS & 150DAS & 180DAS \\
\hline $\mathbf{T}_{1}$ & 7.33 & 9.40 & 9.42 & 11.25 & 13.50 & 5.42 & 7.00 & 9.17 & 10.00 & 10.17 \\
\hline $\mathbf{T}_{2}$ & 7.60 & 9.87 & 11.17 & 13.17 & 16.49 & 7.17 & 7.33 & 10.17 & 10.50 & 11.75 \\
\hline $\mathbf{T}_{\mathbf{3}}$ & 7.80 & 9.80 & 11.42 & 14.08 & 16.33 & 7.42 & 7.67 & 9.83 & 9.58 & 10.67 \\
\hline $\mathbf{T}_{4}$ & 7.53 & 10.60 & 10.42 & 14.83 & 16.58 & 7.58 & 7.25 & 10.67 & 10.75 & 11.33 \\
\hline $\mathbf{T}_{5}$ & 7.33 & 9.60 & 11.92 & 14.83 & 18.54 & 7.50 & 7.92 & 10.17 & 10.33 & 11.50 \\
\hline $\mathbf{T}_{6}$ & 8.67 & 10.13 & 12.08 & 15.58 & 17.25 & 8.00 & 9.83 & 10.83 & 10.75 & 11.75 \\
\hline $\mathbf{T}_{7}$ & 9.47 & 10.80 & 13.75 & 15.83 & 17.72 & 7.75 & 9.00 & 11.33 & 11.97 & 13.42 \\
\hline $\mathbf{T}_{\mathbf{8}}$ & 9.73 & 12.27 & 14.08 & 16.92 & 18.88 & 9.00 & 9.92 & 12.25 & 12.42 & 12.50 \\
\hline $\mathbf{T}_{9}$ & 11.20 & 13.20 & 15.33 & 17.50 & 18.83 & 10.00 & 11.08 & 13.67 & 12.97 & 13.75 \\
\hline $\mathbf{T}_{10}$ & 11.93 & 14.20 & 16.25 & 18.47 & 19.75 & 10.75 & 12.33 & 14.33 & 15.17 & 14.00 \\
\hline F-test & $*$ & $*$ & $*$ & $*$ & $*$ & $*$ & $*$ & $*$ & $*$ & $*$ \\
\hline SEm \pm & 0.78 & 0.49 & 0.36 & 0.53 & 0.91 & 0.73 & 0.39 & 0.50 & 0.89 & 0.66 \\
\hline CD@5\% & 2.30 & 1.45 & 0.93 & 1.55 & 2.69 & 2.16 & 1.15 & 1.49 & 2.61 & 1.94 \\
\hline $\begin{array}{l}* \text { Significant at } 59 \\
\mathrm{~T}_{1}-\text { Soil: Sand: } \\
\mathrm{T}_{2}-\text { Soil: Sand: } \\
\mathrm{T}_{3}-\text { Soil: Sand: } \\
\mathrm{T}_{4}-\text { Soil: Sand: } \mathrm{F} \\
\mathrm{T}_{5}-\text { Soil: Sand: } \mathrm{F}\end{array}$ & $\begin{array}{l}\text { NS - Non } \\
\text { M [2:1:1] }(C \\
\text { micompost } \\
\text { o peat }[2: 1 \\
\text { M: Vermico } \\
\text { M: Cocopea }\end{array}$ & $\begin{array}{l}\text { ificant I } \\
\text { trol) } \\
1: 1] \\
\text { ost }[2: 1: 1: 1 \\
: 1: 1: 1]\end{array}$ & $\begin{array}{r}\mathrm{AS}-\text { Days afte } \\
\mathrm{T}_{6}- \\
\mathrm{T}_{7}- \\
\mathrm{T}_{8}- \\
\mathrm{T}_{9}- \\
\mathrm{T}_{10}\end{array}$ & $\begin{array}{l}\text { sowing } \\
\text { Soil: Sand: F } \\
\text { Soil: Sand: F } \\
\text { oil: Sand: Vc } \\
\text { Soil: Sand: C } \\
\text { Soil: Sand: F }\end{array}$ & $\begin{array}{l}\text { here, PGPR } \\
\text { M: Vermicon } \\
\text { M: PGPR [2: } \\
\text { micompost: } \\
\text { copeat: PGPF } \\
\text { M: Vermico }\end{array}$ & $\begin{array}{l}\text { Microbial cc } \\
\text { ost: Cocope } \\
1: 1] \\
\text { PR [2:1:1:1 } \\
2: 1: 1: 1] \\
\text { post: Cocop }\end{array}$ & ortium ( 25 & I/ polybag) & & \\
\hline
\end{tabular}


Table.4 Effect of different organic mixtures on stem diameter $(\mathrm{cm})$ of seedlings at different stages of growth under polyhouse and nethouse conditions

\begin{tabular}{|c|c|c|c|c|c|c|c|c|c|c|}
\hline \multirow{3}{*}{ Treatment } & \multicolumn{10}{|c|}{ Stem diameter (cm) } \\
\hline & \multicolumn{5}{|c|}{ Polyhouse } & \multicolumn{5}{|c|}{ Nethouse } \\
\hline & 60DAS & 90DAS & 120DAS & 150DAS & 180DAS & 60DAS & 90DAS & 120DAS & 150DAS & 180DAS \\
\hline $\mathbf{T}_{1}$ & 0.46 & 0.52 & 0.68 & 0.74 & 0.78 & 0.43 & 0.46 & 0.54 & 0.63 & 0.70 \\
\hline $\mathbf{T}_{2}$ & 0.46 & 0.58 & 0.70 & 0.76 & 0.85 & 0.43 & 0.47 & 0.57 & 0.68 & 0.73 \\
\hline $\mathbf{T}_{\mathbf{3}}$ & 0.43 & 0.60 & 0.75 & 0.81 & 0.90 & 0.44 & 0.52 & 0.62 & 0.69 & 0.79 \\
\hline $\mathbf{T}_{4}$ & 0.44 & 0.66 & 0.73 & 0.83 & 0.91 & 0.45 & 0.53 & 0.65 & 0.71 & 0.82 \\
\hline $\mathbf{T}_{5}$ & 0.46 & 0.66 & 0.78 & 0.81 & 0.92 & 0.43 & 0.57 & 0.64 & 0.75 & 0.80 \\
\hline $\mathbf{T}_{6}$ & 0.45 & 0.67 & 0.73 & 0.81 & 0.93 & 0.44 & 0.62 & 0.68 & 0.74 & 0.81 \\
\hline $\mathbf{T}_{7}$ & 0.45 & 0.68 & 0.77 & 0.84 & 0.92 & 0.44 & 0.65 & 0.67 & 0.76 & 0.81 \\
\hline $\mathbf{T}_{8}$ & 0.48 & 0.69 & 0.79 & 0.87 & 0.94 & 0.45 & 0.64 & 0.72 & 0.75 & 0.84 \\
\hline $\mathbf{T}_{9}$ & 0.49 & 0.71 & 0.83 & 0.91 & 1.01 & 0.48 & 0.68 & 0.72 & 0.79 & 0.89 \\
\hline $\mathbf{T}_{10}$ & 0.55 & 0.75 & 0.87 & 0.95 & 1.07 & 0.53 & 0.67 & 0.77 & 0.85 & 0.92 \\
\hline F-test & NS & $*$ & $*$ & $*$ & $*$ & NS & $*$ & $*$ & $*$ & $*$ \\
\hline SEm \pm & 0.04 & 0.02 & 0.03 & 0.02 & 0.03 & 0.02 & 0.02 & 0.02 & 0.02 & 0.024 \\
\hline CD@5\% & 0.11 & 0.07 & 0.07 & 0.06 & 0.10 & 0.06 & 0.07 & 0.06 & 0.06 & 0.07 \\
\hline $\begin{array}{l}* \text { Significant } \\
\mathrm{T}_{1}-\text { Soil: } \mathrm{Sar} \\
\mathrm{T}_{2}-\text { Soil: } \mathrm{Sar} \\
\mathrm{T}_{3}-\text { Soil: } \mathrm{Sar} \\
\mathrm{T}_{4}-\text { Soil: } \mathrm{Sar} \\
\mathrm{T}_{5}-\text { Soil: } \mathrm{Sar}\end{array}$ & $\begin{array}{l}5 \% \text { NS }-\mathrm{N} \\
\text { FYM [2:1: } \\
\text { Vermicom } \\
\text { Coco peat } \\
\text { FYM: Ver } \\
\text { FYM: Coc }\end{array}$ & $\begin{array}{l}\text { Significan } \\
\text { (Control) } \\
\text { [ }[2: 1: 1] \\
1: 1] \\
\text { compost }[2 \text { : } \\
\text { eat }[2: 1: 1: 1\end{array}$ & DAS - & $\begin{array}{l}\text { S after sowin } \\
- \text { Soil: Sand } \\
\text { - Soil: Sand } \\
\text { - Soil: Sand: } \\
\text { - Soil: Sand } \\
\text { - Soil: San }\end{array}$ & $\begin{array}{l}\text { Where, PG] } \\
\text { YM: Vermicc } \\
\text { YM: PGPR [ } \\
\text { rmicompost: } \\
\text { ocopeat: PGI } \\
\text { YM: Vermic }\end{array}$ & $\begin{array}{l}\text { - Microbi } \\
\text { post: Coco } \\
: 1: 1] \\
\text { JPR [2:1:1 } \\
\text { [2:1:1:1] } \\
\text { ipost: Cocc }\end{array}$ & $\begin{array}{l}\text { onsortium } \\
\text { t }[2: 1: 1: 1: 1\end{array}$ & $\mathrm{ml} /$ polybag & & \\
\hline
\end{tabular}


Table.5 Effect of different organic mixtures on vigour index of fresh weight and dryweight of seedlings at 180 DAS under polyhouse and nethouse conditions

\begin{tabular}{|c|c|c|c|c|}
\hline \multirow[b]{2}{*}{ Treatment } & \multicolumn{2}{|c|}{ Polyhouse } & \multicolumn{2}{|c|}{ Nethouse } \\
\hline & $\begin{array}{c}\text { Vigour index of fresh } \\
\text { weight }\end{array}$ & $\begin{array}{c}\text { Vigour index of dry } \\
\text { weight }\end{array}$ & $\begin{array}{c}\text { Vigour index of fresh } \\
\text { weight }\end{array}$ & $\begin{array}{c}\text { Vigour index of dry } \\
\text { weight }\end{array}$ \\
\hline$T_{1}$ & 1649.50 & 1099.00 & 1355.57 & 917.73 \\
\hline $\mathbf{T}_{2}$ & 1857.83 & 1258.75 & 1540.73 & 1078.42 \\
\hline $\mathbf{T}_{3}$ & 2314.77 & 1593.58 & 1945.50 & 1381.83 \\
\hline $\mathbf{T}_{4}$ & 2090.83 & 1460.50 & 1867.67 & 1360.63 \\
\hline $\mathbf{T}_{5}$ & 2609.00 & 1815.33 & 2077.27 & 1531.83 \\
\hline$T_{6}$ & 2664.57 & 1911.08 & 1998.60 & 1491.67 \\
\hline $\mathbf{T}_{7}$ & 2479.08 & 1804.27 & 2484.90 & 1842.60 \\
\hline $\mathbf{T}_{8}$ & 2972.67 & 2156.17 & 2402.53 & 1783.92 \\
\hline $\mathbf{T}_{9}$ & 3377.48 & 2458.50 & 2644.70 & 1992.30 \\
\hline$T_{10}$ & 3822.00 & 2854.60 & 3051.60 & 2286.43 \\
\hline $\mathbf{F}$ - test & $*$ & $*$ & * & $*$ \\
\hline SEm \pm & 141.42 & 106.13 & 148.526 & 115.71 \\
\hline CD@5\% & 417.18 & 313.08 & 438.152 & 341.33 \\
\hline \multicolumn{3}{|c|}{$*$ Significant at $5 \% \quad$ DAS - Days after sowing } & oil: Sand: FYM [2:1:1] ( & ontrol) \\
\hline \multicolumn{2}{|c|}{$\mathrm{T}_{2}-$ Soil: Sand: Vermicompost [2:1:1] } & $\mathrm{T}_{3}-$ & oil: Sand: Cocopeat $[2: 1$ & \\
\hline \multicolumn{5}{|c|}{$\mathrm{T}_{4}-$ Soil: Sand: FYM: Vermicompost [2:1:1:1] } \\
\hline \multicolumn{5}{|c|}{$\mathrm{T}_{6}-$ Soil: Sand: FYM: Vermicompost: Cocopeat [2:1:1:1:1] } \\
\hline \multicolumn{5}{|c|}{$\mathrm{T}_{8}-$ Soil: Sand: Vermicompost: PGPR [2:1:1:1] } \\
\hline \multicolumn{5}{|c|}{$\mathrm{T}_{10}-$ Soil: Sand: FYM: Vermicompost: Cocopeat: PGPR [2:1:1:1:1:1] } \\
\hline
\end{tabular}

Table.6 Effect of different organic mixtures on root length of seedlings (cm) at 180 DAS under polyhouse and nethouse conditions

\begin{tabular}{|c|c|c|}
\hline Treatment & $\begin{array}{c}\text { Polyhouse } \\
\text { Root length(cm) }\end{array}$ & $\begin{array}{c}\text { Nethouse } \\
\text { Root length(cm) }\end{array}$ \\
\hline $\mathbf{T}_{\mathbf{1}}$ & 24.23 & 22.20 \\
\hline $\mathbf{T}_{\mathbf{2}}$ & 24.61 & 23.82 \\
\hline $\mathbf{T}_{\mathbf{3}}$ & 32.62 & 26.50 \\
\hline $\mathbf{T}_{\mathbf{4}}$ & 28.82 & 28.78 \\
\hline $\mathbf{T}_{\mathbf{5}}$ & 34.18 & 29.07 \\
\hline $\mathbf{T}_{\mathbf{6}}$ & 38.24 & 30.15 \\
\hline $\mathbf{T}_{\mathbf{7}}$ & 31.87 & 32.10 \\
\hline $\mathbf{T}_{\mathbf{8}}$ & 32.32 & 32.95 \\
\hline $\mathbf{T}_{\mathbf{9}}$ & 39.99 & 34.65 \\
\hline $\mathbf{T}_{\mathbf{1 0}}$ & 41.54 & 35.74 \\
\hline $\mathbf{F}-\mathbf{t e s t}$ & $*$ & $*$ \\
\hline SEm \pm & 1.22 & 0.92 \\
\hline $\mathbf{C D} @ \mathbf{5 \%}$ & 3.59 & 2.74 \\
\hline
\end{tabular}

*Significant at $5 \% \quad$ DAS - Days after sowing $\mathrm{T}_{2}$ - Soil: Sand: Vermicompost [2:1:1]

$\mathrm{T}_{4}$ - Soil: Sand: FYM: Vermicompost [2:1:1:1]

$\mathrm{T}_{6}$ - Soil: Sand: FYM: Vermicompost: Cocopeat [2:1:1:1:1] $\quad \mathrm{T}_{7}-$ Soil: Sand: FYM: PGPR [2:1:1:1]

$\mathrm{T}_{8}-$ Soil: Sand: Vermicompost: PGPR [2:1:1:1]

$\mathrm{T}_{1}-$ Soil: Sand: FYM [2:1:1] (Control)

$\mathrm{T}_{3}$ - Soil: Sand: Cocopeat [2:1:1]

$\mathrm{T}_{5}$ - Soil: Sand: FYM: Cocopeat [2:1:1:1]

$\mathrm{T}_{10}$ - Soil: Sand: FYM: Vermicompost: Cocopeat: PGPR [2:1:1:1:1:1]

Where, PGPR - Microbial consortium ( $25 \mathrm{ml} /$ polybag) 


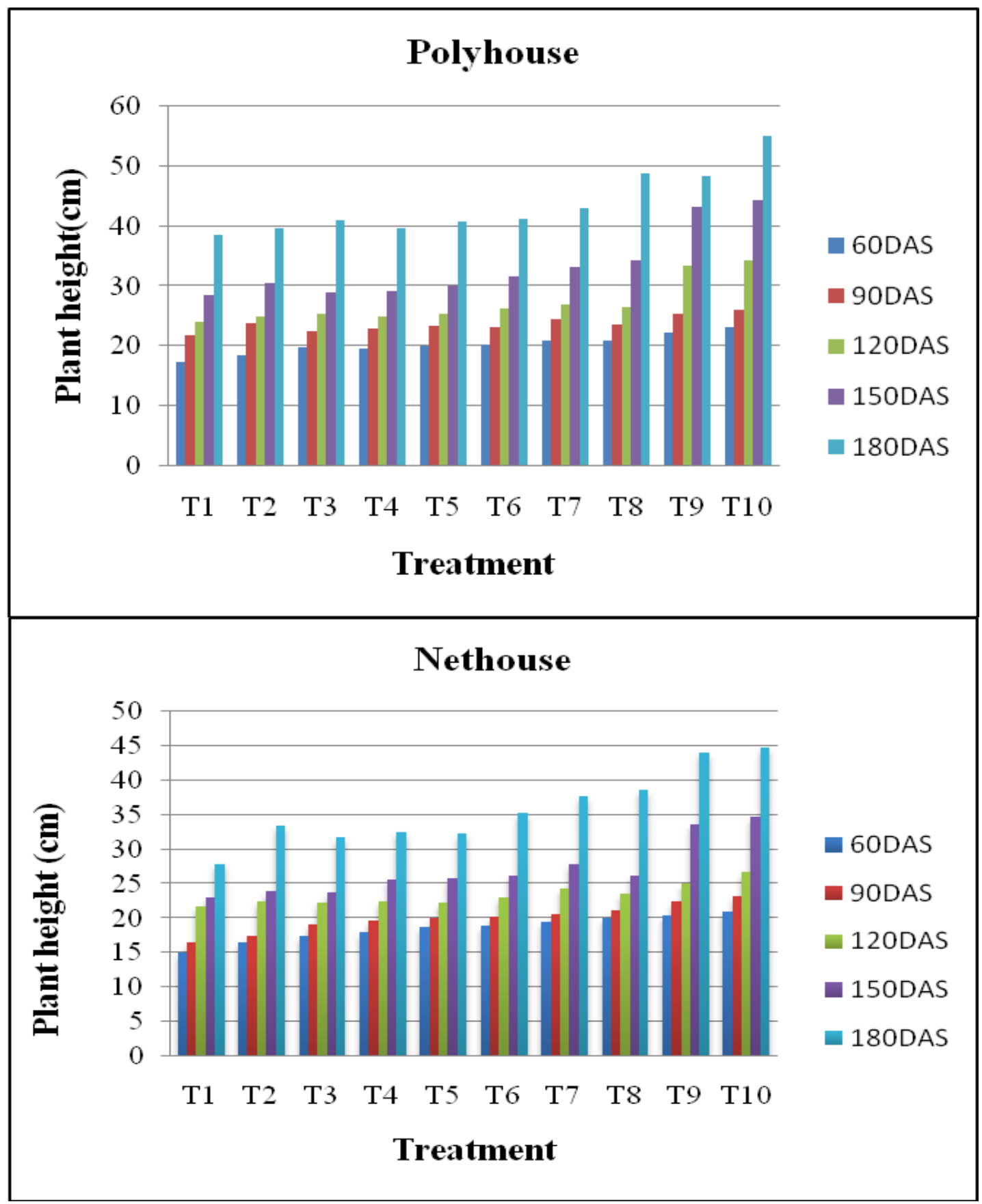

Fig.1 Effect of different organic mixtures on plant height $(\mathrm{cm})$ at different stages of growth under polyhouse and nethouse condition

$\mathrm{T}_{1}$ - Soil: Sand: FYM [2:1:1] (Control)

$\mathrm{T}_{2}$ - Soil: Sand: Vermicompost [2:1:1]

$\mathrm{T}_{3}-$ Soil: Sand: Cocopeat $[2: 1: 1]$

$\mathrm{T}_{4}$ - Soil: Sand: FYM: Vermicompost [2:1:1:1]

$\mathrm{T}_{5}-$ Soil: Sand: FYM: Cocopeat $[2: 1: 1: 1]$
$\mathrm{T}_{6}-$ Soil: Sand: FYM: Vermicompost: Cocopeat [2:1:1:1:1]

$\mathrm{T}_{7}$ - Soil: Sand: FYM: PGPR [2:1:1:1]

$\mathrm{T}_{8}$ - Soil: Sand: Vermicompost: PGPR [2:1:1:1]

$\mathrm{T}_{9}-$ Soil: Sand: Cocopeat: PGPR [2:1:1:1]

$\mathrm{T}_{10}$ - Soil: Sand: FYM: Vermicompost: Cocopeat: PGPR

$[2: 1: 1: 1: 1: 1]$ 


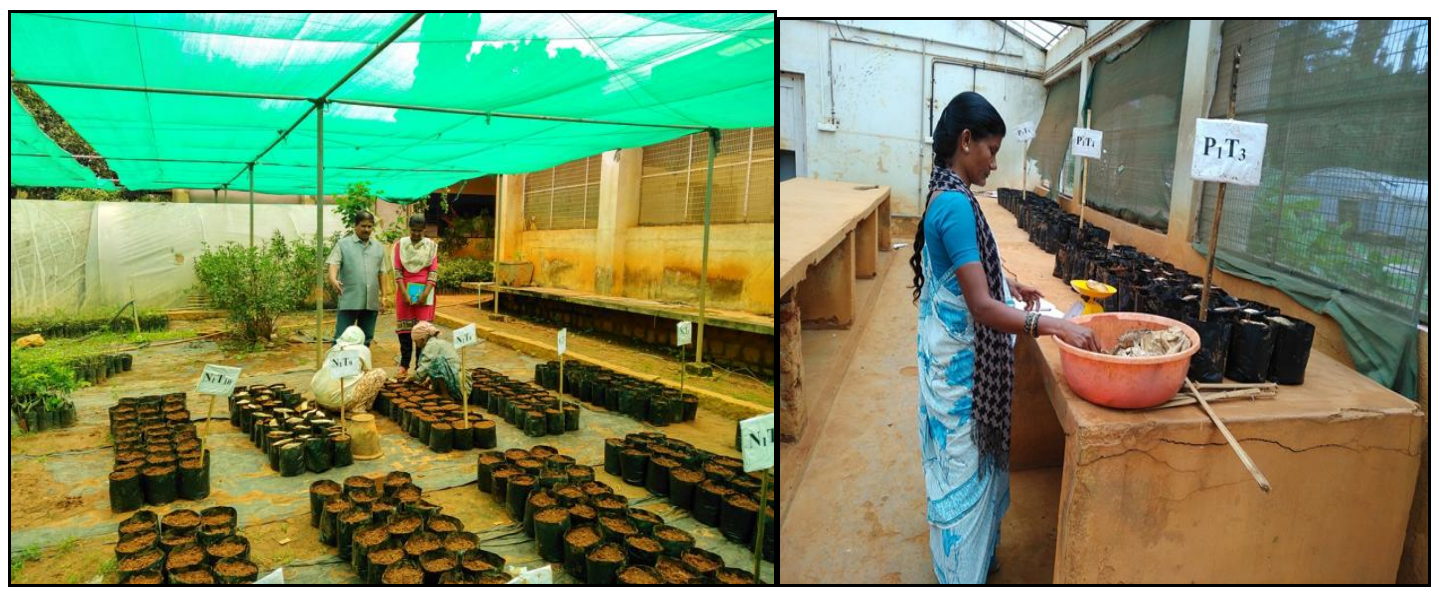

Plate.1 Sowing of mango stones under nethouse and polyhouse conditions

The reason for highest seedling vigour index may be due to the portion of the cocopeat added to the medium helped in meeting immediate requirement of nutrients, along with availability of balanced nutrition and optimum quantity of plant growth promoting rhizobacteria, that helps in nutrient and water uptake and also maintenance of good physical and chemical properties of the media (Vasantha et al., 2014) in tamarind.

\section{Root length $(\mathrm{cm})$ at 180 DAS}

The root lengths of mango seedlings were significantly varied with the age of seedlings among the treatments (Table 6) under polyhouse and nethouse conditions. Significant differences on root length was found among the treatments under polyhouse condition.

The highest root length $(41.54 \mathrm{~cm})$ was recorded in the treatment $\left(\mathrm{T}_{10}\right)$ combined with Soil: Sand: FYM: Vermicompost: Cocopeat: PGPR [2:1:1:1:1:1] which was also found to be on par in $\mathrm{T}_{9}(39.99 \mathrm{~cm})$ with Soil: Sand: FYM: Cocopeat: PGPR [2:1:1:1:1] and the lowest root length $(24.23 \mathrm{~cm})$ was noticed in the control treatment $\left(\mathrm{T}_{1}\right)$ with only basal media at 180 DAS. Similarly, under nethouse conditions, a significant difference on root length was found among the treatments. The highest root length $(35.74 \mathrm{~cm})$ was recorded in the treatment $\left(\mathrm{T}_{10}\right)$ consists of Soil: Sand: FYM: Vermicompost: Cocopeat: PGPR [2:1:1:1:1:1] which was also found to be on par in $\mathrm{T}_{9}(34.65 \mathrm{~cm})$ with Soil: Sand: Cocopeat: PGPR [2:1:1:1] and the lowest root length $(22.20 \mathrm{~cm})$ was noticed in the control treatment $\left(T_{1}\right)$ at 180 DAS.

The highest root length per plant may be due to the organic mixtures with plant growth promoting substances are attributable to enhance the lateral root length and deeper penetration of roots in treatment containing mycorrhizal infection. A larger root mass was reported to accompany mycorrhizal infections (Awasthi et al., 1998). A whole mycorrhizal root system would be expected to be increased because of such greater assimilation of carbon in peach seedlings.

An investigation was carried out on effect of different organic mixtures on stone germination and seedling growth of mango cv. Totapuri under different growing conditions (polyhouse and nethouse condition). Based on results obtained in this study, it may be concluded that the treatment consists of soil: sand: FYM: vermicompost: cocopeat: PGPR [2:1:1:1:1:1] was found be better compared to all the treatment combinations under polyhouse and nethouse conditions. All the growth parameters of mango cv. Totapuri were found to be superior in the treatment consists of soil: sand: FYM: vermicompost: cocopeat: PGPR [2:1:1:1:1:1] under polyhouse condition compared to nethouse conditions. 


\section{References}

Awasthi, R. P., Godara, R. K. and Kaith, N.S., 1998, Interaction effect of mycorrhizae and Azotobacter inoculation on micronutrient uptake by peach seedlings.Indian J. Hort., 11: 1-5.

Chiranjeevi, M. R., Hongal, S., Vinay, G. M., Muralidhara, B. M. and Sneha, M. K., 2018, Influence of media and biofertilizers on seed germination and seedling vigour of Aonla. Int. J. Curr. Microbiol. App. Sci., 7(1): 587-593.

De Candolle., 1904, The origin of cultivated plants. Cambridge University Press.

Fisher, R. A., 1963, Statistical tables for biological, agricultural and medical research Longman group Ltd., England.

Geetha, R., Selvakumari, A. and Sujatha, K., 2007, Evaluation of mango stones under nursery conditions. Pl. Arch., 7(2): 697698.

Jayantraman. 2012, Response of Azotobacter, Pseudomonas and Trichoderma on growth of apple seedling, International Conference on Biological and Life Sciences IPCBEE, 40: 83-90.

Jayashree, C. and Jagadeesh, K. S., 2017, Testing the Effect of the Microbial Consortium on Growth of Vegetable Seedlings in a Farmer's Nursery. Int. J. Curr. Microbiol. App. Sci., 6(2): 16361639.

Pathak, V. K. and Singh, S. and Saini, R. S., 2013, Impact of bio inoculants on seed germination and plant growth of guava (Psidium guajava L.). J. Hort. Agroforestry., 10(5): 183-185.

Parameshwari, K., Srimathi, P. and Malarkodi.
2001, Influence of biofertizer pelletization on elite seedling production of tamarind in nursery. Seed Res., 29(1): 58-62.

Sahni, S., Sarma, B. K., Singh, D. P., Singh, H. B. and Singh K. P., 2007 Vermicompost enhances performance of plant growthpromoting rhizobacteria in Cicer arietinum rhizosphere against Sclerotium rolfsii. Crop Protection., 27: 369-376.

Samaddar, H. N. and Chakrabarti, U. 1988. Effect of different rootstocks on Himsagar and Langra. Acta Hort., 231: 220-224.

Sinish, M. S., Mercy, G. and John, P. S., 2005, Organic methods for cashew root stock production. Cashew, 19(1): 8-15.

Sukhjit Kaur., 2017, Effect of growing media mixtures on seed germination and seedling growth of different mango (Mangifera indica L.) Cultivars under submountaineous conditions of punjab. Chem. Sci. Rev. Lett., 6(23): 1599-1603.

Vasantha, P. T., Vijendrakumar, R, C., Guruprasad, T, R., Mahadevamma, M. and Santhosh, K. V., 2014, Studies on effect of growth regulators and biofertilizers on seed germination and seedling growth of tamarind (Tamarindus indica L.) Pl. Arch., 14(1): 155-160.

Venkata Subbaiah, K., Reddy, R. V. S. K., Shali Raju, G., Karunasree, E., Sekhar, V., Nirmala, V. T., Devivaraprasad Reddy, A. and Deepthi, V., 2018, Effect of Different Levels of Arka microbial consortium on seed germination and survival rate in Brinjal cv. Dommeru Local. Int. J. Curr. Microbiol. App. Sci., 7(6): 2821-2825.

\section{How to cite this article:}

Vandana, S., P. Venkatesha Murthy and Balesh Goudappanavar. 2020. Effect of Organic Mixtures on Stone Germination and Seedling Growth of Mango (Mangifera indica) cv. Totapuri under Nethouse and Polyhouse Conditions. Int.J.Curr.Microbiol.App.Sci. 9(04): 1643-1655. doi: https://doi.org/10.20546/ijcmas.2020.904.193 